\title{
Modeling of a landlord-tenant agricultural system in the environmental context
}

\author{
Natali Hritonenko* \\ Department of Mathematics, Prairie View A\&M University, Box 519, Prairie View, TX 77446, USA \\ E-mail: nahritonenko@pvamu.edu \\ Yuri Yatsenko \\ School of Business, Houston Baptist University, 7502 Fondren, Houston, TX 77074, USA \\ E-mail: yyatsenko@hbu.edu \\ Renan-Ulrich Goetz \\ Department of Economics, University of Girona, Campus Montilivi, 17071 Girona, Spain \\ E-mail: renan.goetz@udg.edu \\ Angels Xabadia \\ Department of Economics, University of Girona, Campus Montilivi, 17071 Girona, Spain \\ E-mail: angels.xabadia@udg.edu
}

\begin{abstract}
A hierarchical economic-environmental model is formulated to analyze the sustainable management of farming production in a landlord-tenant system. The problem under study is interdisciplinary and combines various agricultural, economic, social, and environmental factors. To maximize profit, the landlord chooses a rental payment and the duration of a lease contract. Tenants invest into farmyard fertilizer in order to improve future crop growth and maximize their profit. It is shown that the qualitative behavior of optimal trajectories in the landlord-tenant problem is mostly affected by environmental conditions rather than by the end-of-horizon and delay effects. A range of model parameters is identified, where the optimal trajectory coincides with the first-best environmentally and socially efficient solution.
\end{abstract}

Key Words: Developing countries, landlord-tenant relationship, first-best solution, soil quality, holdup problem, optimization, environmental sustainability

1. Introduction. The sustainable management of natural resources requires preserving the resource so that it can be utilized in the future. While owners, when using the resource themselves, intend to preserve it in the long-run, producers, who have leased the resource, are less interested in preserving it towards the end of the leasing period, e.g., Caputo and Lueck (2003). Moreover, the producers can stop investing in

\footnotetext{
* The corresponding author. Natali Hritonenko, Department of Mathematics, Prairie View A\&M University, Box 519, Prairie View, TX 77446, USA, e-mail: nahritonenko@pvamu.edu
} 
the resource and/ or exploit it beyond the sustainable extraction limits as the lease end approaches. This situation is known in economics as a holdup problem. The reason for the holdup problem is that the producer's investments into resource will not be compensated when the lease contract ends. Producers hold up investments since the investment costs cannot be recovered and may be ex-post expropriated by the owner after the contract ends. The expropriation happens when the parties cannot write complete contracts ex ante and investments are not verifiable by a third party. The non-verifiability is also the reason why the resource owner cannot commit to compensate the other party.

In this paper we concentrate on landlord-tenant relations and agricultural soil as an example of the natural resource. Intensive agricultural production and continuous application of mineral fertilizers reduce the soil fertility. Afterwards, the degraded soil favors soil erosion processes, which, in turn, may lead to sedimentation and siltation processes in rivers and lakes and affect negatively the environmental services provided by the waterways. In the case of agricultural land, the landlord either cultivates the land him/herself or leases it out in form of a fixed-rent or sharecropping contract. To maximize the profit, the landlord chooses the rental payment and the duration of the lease contract. Long-term contracts minimize the landlord's transaction cost related to signing new contracts. However, a long-term contract impedes dissolving the contractual relationship if certain external conditions change and advise to end the relationship (e.g., necessity to sell the land). Hence, the landlord has to strike a balance between the benefits of a long term contract and the flexibility of a short term contract.

Starting with a quite general dynamic setting, we focus on a specific agricultural production problem, where tenants invest into organic fertilizer, such as farmyard manure. Farmers need to apply organic fertilizers to improve and maintain soil quality and soil fertility. This is the most essential investment made by tenants in many African and Asian countries (Besley (1995), Jacoby and Mansury (2008)). Yet, the positive effect of manure is not immediate since the nutrients are released slowly, and, thus, the improvement of the soil structure (humus content) is a gradual process over time.

The objective of this paper is to analyze the landlord-tenant relationship and establish conditions that guarantee the sustainable management of agricultural soil. This relationship involves various economic and biophysical processes and challenges caused by opposing interests of the involved parties, unobservable character of the investment into soil fertility, and different types and duration of the landlord-tenant contracts. Given the limited supply of agricultural land, tenants in many part of the world have no bargaining power (Akhter et al (2012), Dubois (2008)), and landlords make a "take it or leave it" offer. We formulate an integrated economic-environmental model and provide its qualitative analysis that leads to some interesting economic upshots and policy recommendations. The important and novel 
features of our model are: (a) it is based on dynamic optimization, (b) both production and investment depend on the environmental variable (soil quality), and (c) the duration of the landlord-tenant contract is endogenous.

A wide strand of the literature on the incomplete contract theory (see Hart and Moore (2007)) analyzes under which conditions the holdup problem arise and how to overcome it. However, only a few papers consider the holdup problem within a dynamic framework. Che and Sakovics (2004), for instance, analyze a dynamic context where two agents invest in one period and negotiate in the subsequent one, in which case the investment decision is only taken once per period. They find that there exists an asymptotically efficient equilibrium where all investments take place at once. Rey and Salanie (1990) use a dynamic framework but concentrate on the adverse selection problem of the landlord and do not consider investments. The setup by Guriev and Kvasov (2005) is close to our work but differs as they do not consider environmental aspects or the delayed effect of the investments. Yet, the most important difference between their work and the present study is the reliance on the third-party enforcement, i.e., they assume that the provision of a service can be verified by a third party. Moreover, since investments in our model may have a delayed impact, the occurrence of a holdup problem seems to be even more likely. Like some previous literature, e.g., Gul (2001), Guriev and Kvasov (2005), Lichtenberg (2007), we first address the question whether and how the socially and environmentally efficient solution (aka the first-best solution) can be achieved. Next, we explore how environmental and economic parameters determine the model optimal dynamics, including the possibility of a holdup problem.

The paper is organized as follows. Section 2 introduces the landlord-tenant problem, provides its interpretation, and discusses investigation techniques. Section 3 studies the tenant problem. Section 4 analyzes the landlord problem. Section 5 addresses the landlord-tenant problem with a delayed impact of the investment on the soil fertility. Section 6 concludes.

2. Economic-Environmental Model and Optimization Problem. Let us consider an agricultural production system that consists of one landlord and a number of identical tenants. The agricultural production negatively affects the soil quality, which can be improved by specific investment into the soil provided by the tenants. This investment is not compensated by the landlord because it cannot be observed and verified. The length of the contracts between the landlord and tenants is assumed to be finite and be chosen endogenously. The landlord chooses the annual rent of the land and the optimal duration of the lease contract in order to maximize the present value of his/ her infinite-horizon profit. We assume that the tenants have no bargaining power so that the landlord is in the position to make a "take it or leave 
it" offer. For the given rent and contract duration, the tenants determine the specific investments that maximize the present value of their profit while the contract is in force.

Our study concentrates on developing countries that practice subsistence agriculture and/or have no access to markets. In such countries, farmers, who are mostly poor, cannot afford to buy mineral fertilizer and rely on the use of manure in order to improve the productivity of the soil. In these cases, the productive input is related to the investment. Under the assumptions of imperfect commitment from both parties, unobservable investment, and the rational economic behavior of both landlord and tenants, their relationship can be described as a two-level hierarchical dynamic optimization problem that involves two interrelated problems: the landlord problem and the tenant problem.

2.1. The problem of the landlord. The landlord determines the optimal rent $R(t), t \in[0, \infty)$, and the contract length $T>0$ that maximize the landlord's discounted profit over the infinite horizon:

$$
I=\sum_{j=0}^{\infty} \int_{j T}^{(j+1) T} e^{-r t} R(t) d t-V(T),
$$

where a given function $V(T)>0$ denotes transaction costs associated with signing a new contract and the expected costs of being trapped in a long-term contract that does not allow adjustment to changes of external conditions. Naturally, these costs depend on the duration of a contract. We assume that the Ushaped function $V(T)$ decreases at small $T$ and increases when $T$ is large, so that it has a unique minimum. For simplicity, the unknown optimal contract length $T$ in (1) is suggested the same for all sequential contracts $j=0,1,2, \ldots$

2.2. The problem of the tenant. For a fixed number $j=0,1,2, \ldots$, a given contract duration $T$, and a given rent $R(t), t \in[j T,(j+1) T]$, the tenant determines the investment $m(t), t \in[j T,(j+1) T]$, that maximizes

$$
I_{j}=\int_{j T}^{(j+1) T} e^{-r t}[B(m(t), s(t))-c m(t)-R(t)] d t
$$

where the "environmental quality" variable $s(t)$ satisfies the integral-differential equation:

$$
s^{\prime}(t)=-h(m(t), s(t))+\int_{t-\Delta}^{t} f(t-\tau) g(m(\tau)) d \tau, \quad t \in[j T,(j+1) T]
$$

under the inequality-constraints 


$$
\begin{gathered}
m(t) \geq 0, \quad s(t) \geq 0, \\
B(m(t), s(t))-c m(t)-R(t) \geq u_{0}>0,
\end{gathered}
$$

and the initial conditions:

$$
\begin{array}{ll}
\text { at } j=0: & m(t)=m_{0}(t), \quad t \in[-\Delta, 0], \quad s(0)=s_{0}, \\
\text { at } j>0: \quad m(t)=m_{j-1}(t), \quad t \in[j T-\Delta, j T], \quad s(j T)=s_{j-1}(j T),
\end{array}
$$

where $\left(m_{j-1}, s_{j-1}\right)$ is the solution over the previous interval $[(j-1) T, j T]$. The parameters $c, \Delta, u_{0}, s_{0}$ and the functions $B, h, g, f$, and $m_{0}$ are given, while the parameter $T$ and the function $R$ are determined from the landlord optimization problem (1). The smooth functions $B(m, s), h(m, s)$, and $g(m)$ increase in $m$ and $s$, $B(m, s)>0, g(m)>0, f(a)>0, h(m, s)>0$.

The agricultural output $B$ in (2) positively depends on the environmental variable (soil quality) $s$ that is improved with manure application. By (3), the soil quality $s$ is negatively affected by the agricultural production through the function $h$ but can be compensated by the relation-specific investment $m$. Specifically, the investment efficiency function $g(m)>0$ describes the improvement of the soil quality $s$ caused by the investment $m$. This improvement occurs with a certain delay over the interval $[t-\Delta, t]$ in accordance with a given delay distribution $f(t)$ (Hritonenko and Yatsenko (2013)). The inequalityconstraint (5) requires that the tenant's profit cannot be smaller than the given tenant's reservation utility $u_{0}>0$.

The nonlinear optimization problem (2)-(6) includes two unknown variables $m$ and $s$. A model of the rational tenant behavior with several decision variables but with no delay is constructed and discussed in Abdulai et al. (2011).

Problem with no delay. Alongside with the problem (1)-(6), we consider its version with no delay. In this case, the delay distribution $f(t)$ is the Dirac-delta function

$$
f(t-\tau)=\delta(t-\tau)
$$

Therefore, the equation (3) becomes an ordinary differential equation

$$
s^{\prime}=-h(m, s)+g(m), \quad s(0)=s_{0},
$$

and the initial condition for $m$ in (6) is not necessary anymore because there is no delay interval $[t-\Delta, t]$. 
2.3. Synopsis of investigation technique. The problem (1)-(6) is a dynamic optimization problem. In our qualitative analysis, we focus on sustainable development. To find a sustainable solution for the landlord, we disregard the initial condition (6) and find a periodic solution of the tenant problem, which is governed by the same dynamics for all $j$.

In anticipation of a possible holdup problem, the optimal dynamics of the tenant may include a decrease of $m(t)$ and/or $s(t)$ over the interval $[(j+1) T-\Delta,(j+1) T]$ towards the end $(j+1) T$ of the planning horizon $[j T,(j+1) T]$. Correspondingly, the optimal rent $R^{*}(t)$ is generally not constant, and thus, we cannot completely exclude time $t$ from the problem. The qualitative behavior of the optimal of $m, s$, and $R$ over $[j T,(j+1) T]$ will be clarified after the analysis of the tenant's problem in Section 3.

Next, we will show that there exists an initial condition $\left(m_{0}, s_{0}\right)$ that delivers a periodic solution $(\tilde{m}, \tilde{s})$ to the tenant's problem that will be the same for all periods. We prove that this solution attracts asymptotically other solutions for different initial conditions. Then, the dynamics of the optimal rent $\tilde{R}(t)$ over $[j T,(j+1) T]$ along the periodic solution $(\tilde{m}, \tilde{s})$ will be the same for all periods $j$. Therefore, we can rewrite (1) that

$$
\tilde{I}(T)=J(T) \sum_{j=0}^{\infty} e^{-r j T}-V(T)=J(T) /\left(1-e^{-r T}\right)-V(T),
$$

where $J(T)=\int_{0}^{T} e^{-r t} \tilde{R}(t) d t$ describes the landlord's discounted total rent over the contract of duration $T$. The exact dynamics of $J(T)$ will depend on the optimal behavior of the tenant. The further maximization of (9) is mathematically and economically quite standard and will be presented in Section 4. We will first analyze the problem with no delay and then extend results to the case with delay in Section 5.

3. Analysis of Tenant Problem. In this section, we study the dynamics of the tenant optimization problem (2)-(6) at $j=0$ for a given function $R$. To do that, we need to obtain optimality conditions first.

3.1. Necessary conditions for an extremum. Let us choose the unknown function $m$ as the independent control (decision) variable, then $s$ is the dependent (state) variable. Let $m \in L^{\infty}[0, T]$, then the state variable $s$ in (2)-(6) is a.e. differentiable on [0,T], see, e.g., Corduneanu (1991) or Hritonenko and Yatsenko (2013). The inequality $m \geq 0$ of (4) is a standard constraint on the control $m$, while $s \geq 0$ in (4) 
and the inequality (5) are state constraints. For clarity, we provide a standard treatment of the optimization problem (2)-(6) assuming a priori that the state inequality-constraints are inactive. Indeed, Theorem 1 will confirm that $s>0$, while the minimal tenant's profit constraint (5) is not relevant in this section because the rent $R$ will be determined in the landlord problem in Section 4 . Then, we can derive the following maximum principle for the optimization problem (2)-(6).

Lemma 1. Let $m^{*}(t), t \in[0, T]$, be a solution of the optimization problem (2)-(4),(6), then

$$
I^{\prime}(t) \leq 0 \quad \text { at } m *(t)=0, \quad I^{\prime}(t)=0 \text { at } m(t)>0,
$$

where ${ }^{\dagger}$

$$
\begin{aligned}
I^{\prime}(t) & =e^{-r t}\left[B_{m}\left(m^{*}(t), s^{*}(t)\right)-c-h_{m}\left(m^{*}(t), s^{*}(t)\right) \lambda(t)\right. \\
& \left.+g_{m}\left(m^{*}(t)\right) \int_{t}^{\min (T, t+\delta)} e^{-r(u-t)} f(u-t) \lambda(u) d u\right]
\end{aligned}
$$

the state variable $s^{*}(t)$ is determined from (3), and the dual variable $\lambda(t)$ is determined from the dual equation

$$
\lambda^{\prime}(t)=h_{s}\left(m^{*}(t), s^{*}(t)\right) \lambda(t)+r \lambda(t)-B_{s}\left(m^{*}(t), s^{*}(t)\right),
$$

with the transversality condition $\lambda(T)=0$.

Proof is based on perturbation techniques of the optimization theory and the method of Lagrange multipliers applied to integral equations, see, e.g., Corduneanu (1991), Sethi and Thompson (2000), Hritonenko and Yatsenko $(2005,2013)$.

The solution of the dual equation (12) with the initial condition $\lambda(T)=0$ under given $m^{*}$ and $s^{*}$ is

$$
\lambda(t)=\int_{t}^{T} e^{-\int_{t}^{u}\left[r+h_{s}\left(m^{*}(\xi), s^{*}(\xi)\right)\right] d \xi} B_{s}\left(m^{*}(u), s^{*}(u)\right) d u .
$$

By Lemma 1, the interior solution $m^{*}>0, s^{*}>0$ of the tenant problem satisfies $I_{m}{ }^{\prime}(t)=0$ and, therefore, is determined from the system of the nonlinear equations (3), (13), and

\footnotetext{
${ }^{\dagger}$ Here and thereafter, $f_{m}$ denotes the partial derivative of $f$ in $m$.
} 


$$
B_{m}\left(m^{*}(t), s^{*}(t)\right)-c-h_{m}\left(m^{*}(t), s^{*}(t)\right) \lambda(t)+g_{m}\left(m^{*}(t)\right) \int_{t}^{\min (T, t+\Delta)} e^{-r(u-t)} f(u-t) \lambda(u) d u=0
$$

3.2. Tenant problem with no delay: interior trajectories. Our next step is a qualitative study of the problem using economic standard tools, such as the balanced growth and comparative static analysis. In order to proceed with our study, we make more specific assumptions about the employed functions. Here and thereafter in this paper, we focus on the case when: (a) the agricultural output is described by a concave quadratic production function, (b) the negative impact of production on the soil quality is linear; (c) the improvement of the soil quality due to direct application of farmyard manure is also linear. Specifically, we suppose that:

$$
\begin{aligned}
& B(m, s)=Y(m, s)=\left(\alpha m-\beta \frac{m^{2}}{2}\right) s, \\
& h(m, s)=\mu Y(m, s), \quad g(m)=g_{0} m,
\end{aligned}
$$

where $\alpha$ is the agricultural output that corresponds to one unit of the manure (for a given level of the soil quality $s$ in the soil), and $\beta$ describes the diminishing returns of the investment in manure. In practice, the nonlinearity in (15) is usually weak, i.e., the value $\beta$ is much smaller than the value $\alpha$. We will explore this situation in more details later on. Let us measure the soil quality $s$ by the amount of nitrogen per unit of soil (nitrogen pool). Then, $\mu$ is the unit expenditure of nitrogen to produce $1 \$$ of the agricultural output, $g_{0}$ is the increase of nitrogen in soil caused by one unit of manure, $c$ in (2) is the specific cost of one unit of manure. Correspondingly, the dual variable $\lambda$ in (12) has a natural interpretation as the future value of an increase in soil quality (the shadow price of an additional unit of nitrogen in soil).

In the next two sections we analyze the problem (2),(4),(8) with no delay and consider the case with delay in Section 5. In the no-delay case, the equation (14) under condition (7) and specifications (15)-(16) yields

$$
(\alpha-\beta m(t)) s(t)(1-\mu \lambda(t))-c+g_{0} \lambda(t)=0 .
$$

The system (8),(12),(17) for possible interior solution $(m(t), s(t), \lambda(t)), t \in[0, T]$, leads to

$$
m(t)=\frac{\alpha}{\beta}-\frac{c-g_{0} \lambda(t)}{\beta(1-\mu \lambda(t)) s(t)},
$$




$$
\begin{gathered}
s^{\prime}(t)=-\mu\left(\alpha m(t)-\beta m^{2}(t) / 2\right) s(t)+g_{0} m(t), \quad s(0)=s_{0}, \\
\left.\lambda^{\prime}(t)=\mu\left(\alpha m(t)-\beta m^{2}(t)\right) / 2\right) \lambda(t)+r \lambda(t)-\alpha m(t)+\beta m^{2}(t) / 2, \quad \lambda(T)=0 .
\end{gathered}
$$

The solutions $s$ and $\lambda$ to the linear ordinary differential equations (19) and (20) for a given $m$ are

$$
\begin{gathered}
s(t)=g_{0} \int_{0}^{t} e^{-\int_{u}^{t} \mu\left(\alpha m(\xi)-\beta m^{2}(\xi) / 2\right) d \xi} m(u) d u+s_{0} e^{-\int_{0}^{t} \mu\left(\alpha m(\xi)-\beta m^{2}(\xi) / 2\right) d \xi} \\
\lambda(t)=\int_{t}^{T} e^{-\int_{t}^{u}\left(r+\mu\left(\alpha m(\xi)-\beta m^{2}(\xi) / 2\right)\right) d \xi}\left(\alpha m(u)-\beta m^{2}(u) / 2\right) d u
\end{gathered}
$$

The full system (18)-(20) still remains nonlinear with respect to $m$. However, because we have now only one nonlinear function $Y(m, s)$ defined by (15), we can determine meaningful ranges of model parameters that support an interior solution of the optimization problem (2),(4),(8).

Theorem 1. Let ( $m, s, \lambda)$ be a solution of the nonlinear differential equations (18)-(20). Then:

(i) The soil quality $s(t)$ is always positive: $s>0$.

(ii) If $c<g_{0} / \mu$ and $s_{0} \geq g_{0} / \mu \alpha$, then the investment $m(t)$ into soil quality is positive: $m>0$.

(iii) The future value $\lambda(t)$ of the unit increase in the soil quality is always smaller than $1 / \mu: \lambda<1 / \mu$.

Proof. The property (i) is evident by the formula (20). Next, by (22), at $r>0$ we obtain

$$
\left.\lambda(t)<\int_{t}^{T} e^{\left.-\int_{t}^{u} \mu\left(\alpha m(\xi)-\beta m^{2}(\xi)\right) / 2\right) d \xi}\left(\alpha m(u)-\beta m^{2}(u)\right) / 2\right) d u=\frac{1}{\mu}\left[1-e^{\left.-\int_{t}^{T} \mu\left(\alpha m(\xi)-\beta m^{2}(\xi)\right) / 2\right) d \xi}\right]<\frac{1}{\mu}
$$

Thus, property (iii) is true.

Finally, $\quad m(t)=\frac{\alpha}{\beta}-\frac{1}{\beta s(t)}\left[\frac{g_{0}}{\mu}+\frac{c-g_{0} / \mu}{1-\mu \lambda(t)}\right] \quad$ by $\quad(18) . \quad$ At $\quad c<g_{0} / \mu, \quad$ we $\quad$ have $m(t)>\frac{\alpha}{\beta}-\frac{g_{0}}{\beta \mu s(t)}>0$ if $\alpha s(t)>\frac{g_{0}}{\mu}$. Let us show that the last inequality holds under the theorem conditions. Indeed, by (21), we have that

$$
s(t)>g_{0} \int_{0}^{t} e^{-\int_{u}^{t} \mu \alpha m(\xi) d \xi} m(u) d u+s_{0} e^{-\int_{0}^{t} \mu \alpha m(\xi) d \xi}>\frac{g_{0}}{\mu \alpha}\left[1-e^{-\int_{0}^{t} \mu \alpha m(\xi) d \xi}\right]+s_{0} e^{-\int_{0}^{t} \mu o m(\xi) d \xi}>\frac{g_{0}}{\mu \alpha},
$$

if $s_{0} \geq g_{0} / \mu \alpha$. Property (ii) is proven. 
Remark 1. It is worth to mention that, by (21), the soil quality $s(t)$ remains constant on intervals where the investment $m(t)$ is zero, because there is no production without investment by (15). Hence, the holdup problem does not necessary happen in our model in the sense that the soil quality $s(t)$ in (3) is not necessarily smaller for smaller investments $m(t)$. In particular, as follows from the proof of Theorem 1, if the initial soil quality $s(0)=s_{0}$ is larger than $g_{0} / \mu \alpha$, then the soil quality remains larger for any dynamics of $m(t)$.

To properly interpret the conditions of Theorem 1, we provide the dimensional analysis of the model (2)-(6). Let the agricultural output $y$ be in dollars while the nitrogen concentration $s$ be measured in physical units (e.g., kilos) per a unit of soil (e.g., hectare or $\mathrm{m}^{3}$ ). Then we can choose the following units of measurement (UOM) for our model characteristics ${ }^{*}$

$[y]=1000 \$ /$ time - for the agricultural production $y$ per time unit (usually, one year),

$[m]=$ ton/time - for the amount of manure applied $m$ per time unit,

$[s]=$ kilo - for the level of nitrogen $s$ in the soil (nitrogen pool),

$[\mu]=1 / \$$ - for the relative rate $\mu$ of the decrease in nitrogen as a result of the production of $1000 \$ / y e a r$,

$\left[g_{0}\right]=\mathrm{kg} / \mathrm{ton}$ - for the increase of nitrogen pool in the soil as a result of the application of 1 ton of manure,

$[\alpha]=\$ /$ time $/$ ton $/ \mathrm{kg}$ (one dollar per ton of manure/year and per kilo of nitrogen) - for the agriculture output as a result of the application of 1 ton of manure for a given level of soil quality),

$[\beta]=\$ /$ time $/ \operatorname{ton}^{2} / \mathrm{kg} /$ time - for the nonlinearity coefficient $\beta$ in the production function (15),

$[c]=\$ /$ ton- for the specific cost $c$ of 1 ton of manure.

Using these units, we obtain that $[\alpha / \beta]=[m],\left[g_{0}\right]=[c \mu]$, and $[\lambda]=[1 / \mu]$, which will be used in relevant conditions below.

The condition $s_{0} \geq g_{0} / \mu \alpha$ of Theorem 1 is important: if the initial soil quality $s_{0}$ is low, then by (15) the output is also low and does not cover the costs $c$. In this situation, it is optimal to choose $m=0$. Hence the optimal policy is no investment and no production, $m=y=0$, and the soil quality will remain $s_{0}$ forever.

The condition $c<g_{0} / \mu$ of Theorem 1 is even more important and describes the profitability of the manure. It means that the cost per unit of manure $c$ is smaller than the efficiency per unit of manure (the

\footnotetext{
${ }^{*}$ We use the common notation $[x]$ for the UOM of a variable $x$.
} 
increase of agricultural output caused by applying an additional manure unit). These conditions play an important role in our further analysis.

The conditions (ii) are sufficient: the system may have an interior solution even if they do not hold. The system (18), (21), (22) of three nonlinear equations in $m, s$, and $\lambda$ does not allow its general analytic solution. In order to obtain at least an approximate analytic solution of this system, we need to further restrict ourselves to specific cases and ranges of parameter values of the model.

3.3. The case of small investment profitability: an analytic solution. To estimate reasonable ranges of model parameters and develop an approximation technique for the tenant problem (2),(4),(8), let us first analyze a simpler optimization problem of finding the environmentally efficient solution.

3.3.1. Environmentally efficient solution. The environmentally efficient solution of the tenant optimization problem corresponds to an interior steady-state solution of the infinite-horizon case $T=\infty$ of the optimal control problem (2),(4),(8) (Lichtenberg (2007)). This solution describes a situation when the landlord cultivates the land by themselves, which is the most conservative production method (Fraser (2004), Guriev and Kvasov (2005), Rey and Salanie (1990)) as it is in the own interest of the landlord to preserve the resource in the long-run. Jacoby and Mansury (2008) refer to this solution as the first-best solution.

The infinite-horizon version of the optimization problem (2),(4),(8) under assumptions (15)-(16)

$$
\begin{gathered}
\max I_{\infty}=\max \int_{0}^{\infty} e^{-r t}\left[\left(\alpha m-\beta m^{2} / 2\right) s-c m-R\right] d t, \\
s^{\prime}=-\mu\left(\alpha m-\beta m^{2} / 2\right) s+g_{0} m, \quad s(0)=s_{0}, \quad m \geq 0, \quad s \geq 0,
\end{gathered}
$$

does not include time $t$ explicitly. To find a steady-state regime $(\hat{m}, \hat{s}, \hat{\lambda})$ of the optimization problem (23)-(24), we assume the "perfect" initial condition $\hat{s}(0)=s_{0}$. Then, the constant steady-state regime $(\hat{m}$, $\hat{s}, \hat{\lambda})$ exists and is found from the equations (18)-(20) which yield:

$$
\begin{gathered}
\hat{m}=\frac{\alpha}{\beta}-\frac{c-g_{0} \hat{\lambda}}{\beta(1-\mu \hat{\lambda}) \hat{s}}, \\
\hat{s}=\frac{g_{0}}{\mu(\alpha-\beta \hat{m} / 2)},
\end{gathered}
$$




$$
\alpha \hat{m}-\beta \hat{m}^{2} / 2=\frac{r \hat{\lambda}}{1-\mu \hat{\lambda}}
$$

It is challenging to investigate the nonlinear system (25)-(27) in the general case. The analysis below demonstrates that it possesses a positive solution only for some model parameters. In order to better understand the dynamics of the model and obtain an approximate analytic solution, we consider some realistic special cases.

Theorem 2. (On the steady-state first-best regime) Let

$$
0<g_{0}-c \mu<<1, \quad r>\alpha^{2} \mu\left(1-c \mu / g_{0}\right) / \beta
$$

then the nonlinear system (25)-(27) has a unique positive solution $(\hat{m}, \hat{s}, \hat{\lambda})$ given by the approximate formulas

$$
\hat{s} \approx \frac{g_{0}}{\mu \alpha}, \quad \hat{m} \approx m_{\infty}=\frac{\alpha}{\beta}\left(1-\frac{c \mu}{g_{0}}\right) /\left[1-\frac{\alpha^{2} \mu}{r \beta}\left(1-\frac{c \mu}{g_{0}}\right)\right], \quad \hat{\lambda} \approx \frac{\alpha^{2}}{r \beta}\left(1-\frac{c \mu}{g_{0}}\right),
$$

such that $0<\hat{m}<<\alpha / \beta$. If the discount rate $r$ satisfies the stricter inequality

$$
r>>\alpha^{2} \mu\left(1-c \mu / g_{0}\right) / \beta
$$

then the solution (29) is of the form

$$
\hat{s}=\frac{g_{0}}{\mu \alpha}, \quad \hat{m} \approx \frac{\alpha}{\beta}\left(1-\frac{c \mu}{g_{0}}\right), \quad \hat{\lambda} \approx \frac{\alpha^{2}}{\beta r}\left(1-\frac{c \mu}{g_{0}}\right),
$$

such that $\hat{m} \ll \alpha / \beta$ and $\mu \hat{\lambda} \ll 1$.

Here and thereafter, the sign $\approx$ means "with the accuracy up to the first order of the small value $\varepsilon=g_{0} / \mu-c<<1$ and the notation $a<<b$ means that $a / b$ is as small as $\varepsilon$.

Proof. Let us rewrite the equation (25) as $(\alpha-\beta \hat{m}) \hat{s}=\frac{g_{0}}{\mu}-\frac{g_{0} / \mu-c}{1-\mu \hat{\lambda}}$. Substituting $\hat{s}$ from (26), we obtain $\frac{\alpha-\beta \hat{m}}{\alpha-\beta \hat{m} / 2}=1-\left(1-\frac{c \mu}{g_{0}}\right) \frac{1}{1-\mu \hat{\lambda}} \quad$ or $\quad \frac{\beta \hat{m} / \alpha}{2-\beta \hat{m} / \alpha}=\left(1-\frac{c \mu}{g_{0}}\right) \frac{1}{1-\mu \hat{\lambda}}$. 
By Theorem 1, it is natural to assume a priori that the unknown $\hat{\lambda}$ satisfies $\hat{\lambda}<1 / \mu$. Then we have 0 $<\beta \hat{m} / \alpha \ll 1$ provided that the first condition of (28) holds. This inequality allows us to obtain an approximate solution to the nonlinear system (25)-(27). Namely, we can neglect the small term $\beta \hat{m} / \alpha$ in the equations (26) and (27), which leads to $\hat{s} \approx \frac{g_{0}}{\mu \alpha}$ and $\hat{m} \approx \frac{r \hat{\lambda}}{\alpha(1-\mu \hat{\lambda})}$. Combining the last two equations with (25), we obtain the formulas (29) for $\hat{\lambda}$ and $\hat{m}$. The second condition (28) ensures that $\hat{\lambda}<1 / \mu$ indeed.

Finally, one can see that $\mu \hat{\lambda}<<1$ under the condition (30). Thus, by (29), we obtain the formula $\hat{m}=r \hat{\lambda} / \alpha$, i.e., (31) for $\hat{m}$. Theorem 2 is proven.

The proof of Theorem 2 employs a small parameter method. Such methods are used to find approximate analytic solutions and are common in applied sciences. Some successful examples in economics are provided by Bréchet et al. (2013), Hritonenko and Yatsenko (2012), Jovanovic and Yatsenko (2012), Judd and Guu (1997), Khan and Rashid (1982), Santos (1994). The general idea of this method is to reduce the problem under study to a simpler problem with a known solution.

Remark 2. The first constraint $0<g_{0}-c \mu<<1$ in (28) is an important restriction in our analysis. It means that the effectiveness of the investment into the application of manure is higher than its costs. More exactly, the increase in the amount of nitrogen $g_{0}$ in the soil as a result of the application of manure is slightly larger than the associated costs. These are given by the expenditure of a unit of nitrogen $\mu$ to produce one unit of the agricultural output multiplied by the costs of manure $c: g_{0}>\mu c$. In other words, the constraint (28) means that the profitability of the manure application is positive but small compared to the manure investment cost itself: $g_{0}-\mu c<<\mu c$. It can be justified in economic general equilibrium models where the costs of the agricultural output and manure are regulated by the market competition. Here and thereafter, we refer to the difference $g_{0}-\mu c$ as the profitability parameter and call (28) the small investment profitability case.

Remark 3. The second restriction $r>\alpha^{2} \mu\left(1-c \mu / g_{0}\right) / \beta$ of (28) on the discount rate $r$ is rather technical. If it fails (the discount rate is too small compared to $\beta \hat{m} / \alpha$ ), then a positive first-best solution can still exist but it will be described by formulas different from (29)-(31). 
We shall notice that, mathematically, we restrict ourselves to the first-order approximation solution (29) of the nonlinear system (25)-(27) and do not use its higher approximations, because the first-order approximation appears to be more useful in our analysis. Namely, further analysis of the dynamic optimization problem (2)-(6) will use approximations of the same order since its higher-order approximations are not feasible.

3.3.2. Approximate analytic solution of the dynamic tenant problem. Let us return to the full dynamic version (2)-(6) of the tenant problem on the finite horizon $[0, T]$ and find its approximate analytic solution. Based on the outcomes of the previous section, we assume a priori that $|m(t)| \ll \alpha / \beta$, neglect the quadratic terms with $m^{2}$ in the formulas (21)-(22), and obtain

$$
\begin{gathered}
s(t)=\frac{g_{0}}{\mu \alpha}+\left(s_{0}-\frac{g_{0}}{\mu \alpha}\right) e^{-\mu \alpha \int_{0}^{t} m(\xi) d \xi}, \\
\lambda(t)=\int_{t}^{T} e^{-\int_{t}^{u}(r+\mu o m(\xi)) d \xi} \alpha m(u) d u=\frac{1}{\mu}\left[1-e^{-\int_{t}^{T}(r+\mu o m(\xi)) d \xi}\right]-\frac{r}{\mu} \int_{t}^{T} e^{-\int_{t}^{u}(r+\mu o m(\xi)) d \xi} d u \\
=\frac{1}{\mu}-\frac{1}{\mu}\left[e^{-\int_{t}^{T}(r+\mu o m(\xi)) d \xi}+r \int_{t}^{T} e^{-\int_{t}^{u}(r+\mu o m(\xi)) d \xi} d u\right] .
\end{gathered}
$$

Remark 4. Under imposed assumptions, the formula (32) indicates that the optimal $s(t)$ strives to a perfect constant limit that coincides with the first-best soil quality value $\hat{s}=g_{0} / \mu \alpha$. This formula also leads to a rather unexpected conclusion that the qualitative behavior of $s$ is not affected by the dynamics of optimal $m$, at least, if the small profitability condition (28) holds. More exactly, possible and even sharp changes of $m(t)$ would affect the speed of the $s(t)$-convergence to $\hat{s}$ in (32) but they do not change the direction of change (increase or decrease) of $s(t)$, which is determined solely by the difference between $\hat{s}$ and the initial condition $s_{0}$.

The equation (17), $I^{\prime}(t)=0$ for interior trajectories can be presented in the following form:

$$
(\alpha-\beta m(t)) s(t)=\frac{g_{0}}{\mu}+\frac{c-g_{0} / \mu}{1-\mu \lambda(t)} .
$$

The substitution of (32) and (33) into (34) gives 


$$
\begin{aligned}
& -\beta \frac{g_{0}}{\mu \alpha} m(t)-\beta\left(s_{0}-\frac{g_{0}}{\mu \alpha}\right) m(t) e^{-\mu \alpha \int_{0}^{t} m(\xi) d \xi}+\alpha\left(s_{0}-\frac{g_{0}}{\mu \alpha}\right) e^{-\mu \alpha \int_{0}^{t} m(\xi) d \xi}= \\
& =\left(c-\frac{g_{0}}{\mu}\right)\left[e^{-\int_{t}^{T}(r+\mu \alpha m(\xi)) d \xi}+r \int_{t}^{T} e^{-\int_{t}^{u}(r+\mu \alpha m(\xi)) d \xi} d u\right]^{-1} .
\end{aligned}
$$

Under the assumption $|m(t)|<<\alpha / \beta)$ one can notice that $\mu \alpha \int_{0}^{T} m(\xi) d \xi$ is small for a finite $T$. So, using the Taylor series expansion for the exponent in the right-hand part of (35), we obtain

$$
\begin{aligned}
\beta s_{0} m(t) & =\left(\alpha s_{0}-\frac{g_{0}}{\mu}\right)-\alpha\left(s_{0}-\frac{g_{0}}{\mu \alpha}\right) \mu \alpha \int_{0}^{t} m(\xi) d \xi \\
& -\left(c-\frac{g_{0}}{\mu}\right)\left[e^{-\int_{t}^{T}(r+\mu o m(\xi)) d \xi}+r \int_{t}^{T} e^{-\int_{t}^{\mu}(r+\mu a m(\xi)) d \xi} d u\right]^{-1},
\end{aligned}
$$

or

$$
\beta s_{0} m(t)=\left(\alpha s_{0}-\frac{g_{0}}{\mu}\right)-\alpha\left(s_{0}-\frac{g_{0}}{\mu \alpha}\right) \mu \alpha \int_{0}^{t} m(\xi) d \xi-\left(c-\frac{g_{0}}{\mu}\right)[1-\mu \lambda(t)]^{-1}
$$

Applying the Mean Value Theorem to estimate the last integral in the right-hand side of (33), we have

$$
\begin{gathered}
1-\mu \lambda(t)=\left[e^{-\int_{t}^{T}(r+\mu \alpha m(\xi)) d \xi}+\frac{r}{(r+\mu \alpha m(\theta(t)))} \int_{t}^{T} e^{-\int_{t}^{\mu}(r+\mu \alpha m(\xi)) d \xi}(r+\mu \alpha m(u)) d u\right], \text { where } t<\theta(t)<T, \\
\text { or } \quad 1-\mu \lambda(t)=\frac{r}{r+\mu \alpha m(\theta(t))}\left[1-e^{-\int_{t}^{T}(r+\mu \alpha m(\xi)) d \xi}+(r+\mu \alpha m(\theta(t))) e^{-\int_{t}^{T}(r+\mu \alpha m(\xi)) d \xi}\right] .
\end{gathered}
$$

The last expression tends to 1 when $r /[\mu \alpha m(\theta(t))]$ becomes smaller. Therefore, at the condition $r>>$ $\mu \alpha m(t)$ we have $\mu \lambda(t)<<1$, substituting it into (37), we obtain the linear Volterra integral equation of the second kind for $m$ :

$$
m(t)+\frac{\alpha}{\beta}\left(\mu \alpha-\frac{g_{0}}{s_{0}}\right) \int_{0}^{t} m(\xi) d \xi=\frac{1}{\beta}\left(\alpha-\frac{c}{s_{0}}\right)
$$

with the unique solution 


$$
m(t)=\frac{\alpha}{\beta}\left(1-\frac{c}{\alpha s_{0}}\right) e^{-\left(\mu \alpha-g_{0} / s_{0}\right) \alpha t / \beta} .
$$

The function (38) is positive at $\alpha s_{0} \geq c$. If $\alpha s_{0}-c$ is positive and small, then by (38) we have $|m(t)|<<\alpha / \beta$ on the finite interval $[0, T]$. Finally, we see that $r \gg>\mu \alpha m(t)$ if $r>>\mu \alpha\left(\alpha-c / s_{0}\right) / \beta$. The above reasoning is summarized in the following statement.

Theorem 3. If (30) holds and

$$
0 \leq \alpha s_{0}-c<<1
$$

then the optimization problem (2),(4),(8) has a unique interior solution determined by the approximate formulas (32) and (38), such that $\|m\|<<\alpha / \beta$ and $\|\lambda\|<<1 / \mu$, where $\|. .$.$\| denotes the norm in L^{\infty}[0, T]$.

By (32), the optimal soil quality $s(t)$ monotonically increases over the interval $[0, T]$ if the initial value $s_{0}$ is relatively low: $s_{0}<g_{0} / \mu \alpha$, and decreases over $[0, T]$ if $s_{0}>g_{0} / \mu \alpha$.

In the original problem (1)-(6), the final value $s(T)$ of the optimal $s(t)$ on the interval $[0, T]$ is the initial value for the next interval $[T, 2 T]$. Since we are interested in a sustainable solution of the landlord-tenant problem (1)-(6), we need to find the "perfect" initial condition that satisfies $s(0)=s(T)$.

3.3.3. Periodic solution of the tenant problem. By (32), the ideal initial value $s_{0}$ that immediately produces a sustainable solution in the tenant problem (2)-(6) is $s_{0}=g_{0} / \mu \alpha$. Then, the optimal soil quality $s(t)$ is constant over the interval $[0, T]$ and thus satisfies $s(0)=s(T)$.

Theorem 4. If the conditions (28) hold, then the tenant optimization problem (2),(4),(8) has a unique sustainable trajectory

$$
\tilde{s} \approx \frac{g_{0}}{\mu \alpha}, \quad \hat{m} \leq \tilde{m}(t)<m_{\infty}, \quad t \in[0, T]
$$

such that $\tilde{m} \ll \alpha / \beta$,

$$
\tilde{m}(T)=\hat{m} \text { and } \quad \tilde{m}(t) \underset{T-t \rightarrow \infty}{\longrightarrow} m_{\infty},
$$


where $\hat{m}$ is given by $(31)$ and $m_{\infty}$ is given by (29). The trajectory $(\tilde{m}, \tilde{s})$ is an approximate solution to the problem (2)-(6) at $s_{0}=g_{0} / \mu \alpha$. If the discount rate satisfies (30), then the sustainable trajectory is constant:

$$
\tilde{s} \approx \frac{g_{0}}{\mu \alpha}, \quad \tilde{m} \approx \frac{\alpha}{\beta}\left(1-\frac{c \mu}{g_{0}}\right) .
$$

Proof. Substituting $s_{0}=g_{0} / \mu \alpha$ into (35), we obtain the nonlinear integral equation for the corresponding sustainable trajectory $\tilde{m}$ :

$$
\tilde{m}(t)=\frac{\alpha}{\beta}\left(1-\frac{c \mu}{g_{0}}\right)\left[e^{-\int_{t}^{T}(r+\mu a \tilde{m}(\xi)) d \xi}+r \int_{t}^{T} e^{-\int_{t}^{\prime \prime}(r+\mu a \tilde{m}(\xi)) d \xi} d u\right]^{-1} .
$$

The equation (43) is a (non-standard) nonlinear Volterra integral equation of the second kind and, as such, has a unique solution $\tilde{m}(t), t \in[0, T]$ under standard assumptions. Indeed, let us write this equation as $m=A m$, where the operator $A$ is determined by the right-hand side of (43), and consider a small interval $\left[t_{1}, T\right], 0 \leq t_{1}<T$. Then, it is easy to prove that $\left\|A m_{2}-A m_{1}\right\|<\left\|m_{2}-m_{1}\right\|$ and, therefore, the equation (43) has a unique solution $\tilde{m}(t)$ on $\left[t_{1}, T\right]$ for small enough $T-t_{1}$ by the contraction mapping principle (Kolmogorov and Fomin (1975)) also known as the Banach fixed point theorem (Kirk and Khamsi (2001)). Continuing this process for $\left[t_{2}, t_{1}\right], \quad 0 \leq t_{2}<t_{1}$, then for $\left[t_{3}, t_{2}\right]$, and so on, we obtain a unique solution $\tilde{m}(t)$ of $(43)$ over the entire interval $[0, T)$.

To prove the estimate (41) for the solution $\tilde{m}$, we implement the following three steps.

1. Taking the derivative of (43), we obtain that

$$
\begin{aligned}
\tilde{m}^{\prime}(t) & =-\frac{\alpha}{\beta}\left(1-\frac{c \mu}{g_{0}}\right)\left[(r+\mu \alpha \tilde{m}(t)) e^{-\int_{t}^{T}(r+\mu \alpha \tilde{m}(\xi)) d \xi}-r+r(r+\mu \alpha \tilde{m}(t)) \int_{t}^{T} e^{-\int_{t}^{u}(r+\mu \alpha \tilde{m}(\xi)) d \xi} d u\right]^{-1} \\
& \times\left[e^{-\int_{t}^{T}(r+\mu \sigma \tilde{m}(\xi)) d \xi}+r \int_{t}^{T} e^{-\int_{t}^{u}(r+\mu \alpha \tilde{m}(\xi)) d \xi} d u\right]^{-2} .
\end{aligned}
$$

Therefore, $\tilde{m}^{\prime}(t)<0$, at least, at $t=T$ and some $t$ close to $T$.

2. By (43), $\tilde{m}(T)=\hat{m}=\frac{\alpha}{\beta}\left(1-\frac{c \mu}{g_{0}}\right)$ and, hence, $\tilde{m}(t)>\hat{m}$ at some $t$ close to $T$. 
3. Using the last inequality, we have $e^{-\int_{t}^{T}(r+\mu a \tilde{m}(\xi)) d \xi}<e^{-\int_{t}^{T}(r+\mu a \hat{n}) d \xi}$ and by (43)

$$
\tilde{m}(t)<\hat{m}\left[e^{-\int_{t}^{T}(r+\mu \alpha \hat{m}) d \xi}+r \int_{t}^{T} e^{-\int_{t}^{\mu}(r+\mu \alpha \hat{m}) d \xi} d u\right]^{-1}=\hat{m}\left[\frac{r}{r+\mu \alpha \hat{m}}+\frac{\mu \alpha \hat{m}}{r+\mu \alpha \hat{m}} e^{-(r+\mu \alpha \hat{m})(T-t)}\right] .
$$

Therefore, $\tilde{m}(t)<m_{\infty}$ for $t \in[0, T]$ and $\tilde{m}(t)$ approaches $m_{\infty}$ when $T-t \rightarrow \infty$.

Finally, the formula (42) for $\tilde{m}$ for the case $r>>\alpha^{2} \mu\left(1-c \mu / g_{0}\right) / \beta$ is obtained from (40) and (31). The theorem is proven.

Here and thereafter, we refer to the discount rate $r$ as essential if the condition (30) holds. By Theorem 4 , in the case of essential discount rates, the sustainable trajectory (42) of the tenant problem (2),(4),(8) approximately coincides with its "first-best" solution $(\hat{m}, \hat{s})$. By (30), the essential discount rate $r$ is much larger than $\alpha^{2} \mu\left(1-c \mu / g_{0}\right) / \beta$ that includes the product of two small parameters. Indeed, $1-c \mu / g_{0}$ is small by (28), while $\alpha / \beta$ is small because the quadratic nonlinearity (15) should be weak. Therefore, the essential discount rate $r$ in our model can be small, 2-5\% (as usually taken in the economic theory and practice).

4. Analysis of Landlord-Tenant Problem. Following Section 2.1, the landlord's problem is to find the optimal value $T>0$ and the function $R(t) \in[0, \infty)$, that maximize the functional (1):

$$
I=\sum_{j=0}^{\infty} \int_{T j}^{T(j+1)} e^{-r t} R(t) d t-V(T), \text { for a given } V(T) .
$$

Following the technique of Lichtenberg (2007), one can show that the optimal $R(t), t \in[0, \infty)$, will be a corner solution along the inequality-constraint $B(m(t), s(t))-c m(t)-R(t) \geq u_{0}>0$ of (4), i.e., the optimal $R(t)=B(m(t), s(t))-c m(t)-u_{0}$, or

$$
R(t)=\left(\alpha m(t)-\beta m^{2}(t) / 2\right) s(t)-c m(t)-u_{0}, \quad t \in[0, \infty) .
$$

under specifications (15). Thus, finding a sustainable solution to the landlord problem is straightforward if the solution of the tenant problem is known. We consider the simpler case first. 
4.1. Case of essential discount rates. Let us assume that conditions (28) and (30) hold. Then, the tenant problem (2)-(6) has a unique constant sustainable trajectory $(\tilde{m}, \tilde{s})$ which is given by the periodic solution (42) with the perfect initial condition $s_{0}=g_{0} / \mu \alpha$. Along the sustainable trajectory $(\tilde{m}, \tilde{s})$, the optimal sustainable rent $\tilde{R}$ over $[j T,(j+1) T]$ is also constant and is the same for all periods $j$ :

$$
\widetilde{R}_{A}=\frac{\alpha}{\beta}\left(\left(1-\frac{c \mu}{g_{0}}\right)-\frac{1}{2}\left(1-\frac{c \mu}{g_{0}}\right)^{2}\right) \frac{g_{0}}{\mu}-c \frac{\alpha}{\beta}\left(1-\frac{c \mu}{g_{0}}\right)-u_{0} \approx \frac{\alpha}{\beta} \frac{g_{0}}{\mu}\left(1-\frac{c \mu}{g_{0}}\right)^{2}-u_{0}
$$

Remark 5. The trajectory $(\tilde{m}, \widetilde{s})$ is obtained under the condition $c<g_{0} / \mu$ in Section 3. By (45), if the given tenant's reservation utility $u_{0}$ is not zero, $u_{0}>0$, the expense $c$ should be even smaller, namely, $c<\frac{g_{0}}{\mu}\left(1-\sqrt{u_{0} \frac{\beta \mu}{\alpha g_{0}}}\right)$ instead of $c<g_{0} / \mu$ to guarantee that $\tilde{R}_{A}>0$. The stricter inequality for $c$ states that renting the land should be profitable for the landlord.

Then, the total discounted landlord's profit (1) along the sustainable trajectory $(\tilde{m}, \tilde{s})$ is given by

$$
\tilde{I}(T)=\tilde{R}_{A} \sum_{j=0}^{\infty} \int_{j T}^{(j+1) T} e^{-r t} d t-V(T)=\tilde{R}_{A} \int_{0}^{\infty} e^{-r t} d t-V(T) \cong \tilde{R}_{A} / r-V(T) .
$$

Correspondingly, the maximum of $\tilde{I}(T)$ is reached at the same value $T^{*}$ where the minimum of the function $V(T)$ occurs. Thus, the solution of the landlord-tenant problem (1)-(5) is $T^{*}=\operatorname{argmax} V(T)$ and $R^{*} \equiv \widetilde{R}_{A}$.

4.1. Case of general discount rates. Provided that conditions (28) hold, the unique sustainable trajectory $\tilde{s} \approx \hat{s}$ of the problem (2)-(6) is determined by (40) and a slowly decreasing $\tilde{m}(t), t \in[0, T]$, given by (41). Correspondingly, the optimal sustainable rent (44) over $[j T,(j+1) T]$ is the same for all periods $j$ and is also a slowly changing function denoted by $\widetilde{R}_{B}(t)$ :

$$
\begin{gathered}
\tilde{R}_{A}<\tilde{R}_{B}(t)<R_{\infty}, \\
R_{\infty}=\frac{\alpha}{\beta}\left(1-\frac{c \mu}{g_{0}}\right)\left[1-\frac{\alpha^{2} \mu}{r \beta}\left(1-\frac{c \mu}{g_{0}}\right)\right]^{-1}\left(\frac{g_{0}}{\mu}-c\right)-u_{0} .
\end{gathered}
$$


Let us denote the landlord's discounted total rent over the contract of duration $T$ as

$$
J(T)=\int_{0}^{T} e^{-r t} \widetilde{R}_{B}(t) d t
$$

Then, the total infinite-horizon discounted landlord's profit (1) along the sustainable trajectory is

$$
\tilde{I}(T)=J(T) \sum_{j=0}^{\infty} e^{-r j T}-V(T)=J(T) /\left(1-e^{-r T}\right)-V(T)
$$

By the properties of $(\tilde{m}, \tilde{s})$ from Theorem 4 , we can conclude that:

(i). If the contract duration is small: $T \ll<1$, then $\widetilde{R}_{B}(t) \approx \widetilde{R}_{A}$, and, therefore, by (49)-(50),

$$
J(T) \approx \tilde{R}_{A} \int_{0}^{T} e^{-r t} d t \approx \tilde{R}_{A}\left(1-e^{-r T}\right) / r \quad \text { and } \quad \tilde{I}(T) \approx \tilde{R}_{A} / r-V(T)
$$

(ii). If the contract duration is large: $T \gg>1$, then $\widetilde{R}_{B}(t) \approx \widetilde{R}_{\infty}$ on the most part of the interval $[0, T]$, therefore, $J(T) \approx \tilde{R}_{\infty} \int_{0}^{T} e^{-r t} d t \approx \tilde{R}_{\infty}\left(1-e^{-r T}\right) / r \quad$ and $\quad \tilde{I}(T) \approx \tilde{R}_{\infty} / r-V(T)$.

The exact dynamics of $\tilde{I}(T)$ is difficult to determine analytically. By (i) and (ii), we can see that $\tilde{I}(T) \approx W(T)-V(T)$, where $W(T)$ is slowly increasing from $\tilde{R}_{A} / r$ at $T \ll<1$ to $\tilde{R}_{\infty} / r$ at $T \gg 1$. The difference $W(\infty)-W(0)$ is positive and is of the same magnitude as $\left(1-c \mu / g_{0}\right)$, which is small by (28). Therefore, the maximum of $\tilde{I}(T)$ is reached at approximately $T^{*}=\operatorname{argmax} V(T)$.

5. Landlord-Tenant Problem with Delay. To analyze how the delay $\Delta>0$ in the environmental state equation (3) affects the qualitative dynamics of the landlord-tenant problem (1)-(7), we concentrate on the estimate of the differences caused by the delay. Taking into account the challenges that surfaced while studying the problem (1)-(7) with no delay, we restrict ourselves to the small investment profitability case (28). Detailed proofs are omitted due to the space limitation.

5.1. Tenant problem with delay. At the conditions (28), the sought-for functions are slowly changing exponents and an approximate analysis, similar to the one provided in Sections 3 and 4, is possible. 
5.1.1. The environmentally efficient solution. An interior steady-state solution of the infinite-horizon tenant's problem (2)-(6), or according to Lichtenberg (2007) the "first-best" solution appears to be the same as in the case with no delay. Specifically, the infinite-horizon optimization problem

$$
\begin{gathered}
\max I_{\infty}=\max \int_{0}^{\infty} e^{-r t}\left[\left(\alpha m-\beta m^{2} / 2\right) s-c m-R\right] d t, \\
s^{\prime}=-\mu\left(\alpha m-\beta m^{2} / 2\right) s+g_{d} \int_{0}^{\Delta} f(u) m(t-u) d u, \quad m \geq 0, \quad s \geq 0,
\end{gathered}
$$

possesses the constant long-term steady-state regime $(\hat{m}, \hat{s}, \hat{\lambda})$, described by Theorem 2 , at

$$
g_{0}=g_{d} \int_{0}^{\Delta} f(u) d u
$$

Indeed, the state equation in (51) can be rewritten in the form of (19) as $s^{\prime}=-\mu\left(\alpha m-\beta m^{2} / 2\right) s+g_{0} m$, where $g_{0}$ is determined by (52). Thus, Theorem 2 also holds for the problem (1)-(5) with delay.

5.1.2. Approximate "periodic" solution. Let us return to the full dynamic version (2)-(6) of the tenant's problem for a finite planning horizon. Assuming a priori that $|m(t)|<<\alpha / \beta$, we obtain the same explicit formula (33) for $\lambda(t)$. If $m(t)$ is a slowly changing function, then the same approximate formula (32) for $s(t)$ is valid. The difference is in the formula for $m(t)$ which is more complicated than (34) :

$$
m(t)=\frac{\alpha}{\beta}+\frac{1}{\beta[1-\mu \lambda(t)] s(t)}\left[g_{d} \int_{t}^{\min (T, t+\Delta)} e^{-r(u-t)} f(u-t) \lambda(u) d u-c\right]
$$

As in the previous section, we consider separately two cases of different discount rates and start with the simpler case.

Case of essential discount rates: (28) and (30) hold. Similarly to Section 3.2.2, $\mu \lambda(t)<<1$ as $r>>$ $\mu \alpha m(t)$. Hence, when the discount rate is essential and satisfies (30), then the first-order approximation of for $m(t)$ is the same as in (38). In this case, the initial value $s_{0}=g_{0} / \mu \alpha$ delivers the optimal constant soil quality $s(t)$ over the interval $[0, T]$ and, therefore, represents the "periodic" solution of the tenant problem. Thus, the first part of Theorem 4 is also true for the problem with delay: If both (28) and (30) hold, then the tenant problem (2)-(6) with delay has the same unique sustainable trajectory (42) as in the case with no delay. 
General case: (28) holds. The difference between the cases with delay and no delay appears when the discount rate can be small such that condition (30) fails. Remarks 1 and 4 still hold in the case with delay.

Remark 6. The optimal $s(t)$ strives to the first best value $\hat{s}=g_{0} / \mu \alpha$ and the optimal $m(t)$ affects only the speed of this convergence but does not change the direction of the change of $s(t)$. The increase or decrease of $s(t)$ is determined solely by the difference between $\hat{s}$ and the initial condition $s_{0}$. Therefore, in our model (1)-(5) the presence of delay does not cause the decrease of the soil quality at the end of the planning horizon at least if (28) holds.

As before, to analyze a sustainable solution of the landlord-tenant problem (1)-(6), we choose the initial condition $s_{0}=g_{0} / \mu \alpha$ that satisfies $s(0)=s(T)$. The following statement is proven similarly to Theorem 4.

Theorem 5. If (28) holds, then the tenant's problem (2)-(6) with delay has a unique sustainable trajectory $\left(\tilde{s}, \tilde{m}_{d}\right)$, with

$$
\tilde{s}=\frac{g_{0}}{\mu \alpha}, \quad \hat{m} \leq \tilde{m}_{d}(t)<m_{\infty}, \quad t \in[0, T],
$$

such that $\tilde{m}_{d}(T)=\hat{m}$ and $\tilde{m}_{d}(t) \rightarrow m_{\infty}$ at $T-t \rightarrow \infty$.

If the delay function $f(a)$ in (3) is slowly changing: $d f / d a<1$, then the difference

$$
\tilde{m}(t)-\tilde{m}_{d}(t) \approx \begin{cases}0, & 0 \leq t \leq T-\Delta, \\ \alpha \hat{m}(T-t)\left(1-\frac{T-t}{\Delta}\right), & T-\Delta \leq t \leq T\end{cases}
$$

where $\tilde{m}(t)$ is the solution to the problem (2)-(5) with no delay at $g_{0}=g_{d} \int_{0}^{\Delta} f(u) d u$.

Thus, the solution $\tilde{m}_{d}$ with delay in a general case is different from $\tilde{m}$ even in the first-order approximation. The difference $\delta \tilde{m}=\tilde{m}_{d}-\tilde{m}$ determined in Theorem 5 allows us to calculate the reduction of the landlord's rent as a result of the delayed effect of the manure efficiency.

5.2. Landlord problem with delay. In the case (28) of small positive values of the profitability parameter $g_{0}-c \mu$, there are no principal differences in the optimal dynamics between the problem (1)(6) with delay and no delay. 
5.2.1. Case of essential discount rates. Let both (28) and (30) hold. Then, the solution in this case coincides with the no-delay problem of Section 4.1.1. Namely, the optimal sustainable rent $\tilde{R}$ along the constant sustainable trajectory $(\tilde{m}, \tilde{s})$ is constant and given by (45), the total discounted landlord's profit (1) is

$$
\widetilde{I}(T) \approx \widetilde{R}_{A} / r-V(T)=\frac{1}{r}\left[\frac{\alpha}{\beta} \frac{g_{0}}{\mu}\left(1-\frac{c \mu}{g_{0}}\right)^{2}-u_{0}\right]-V(T),
$$

and its maximum is reached at the same value, i.e., $T^{*}=\operatorname{argmax} V(T)$.

5.2.2. Case of general discount rates. Let (28) hold. Then, the tenant problem (2)-(6) with delay has a unique sustainable trajectory: the constant $\tilde{s}$ and a slowly changing function $\tilde{m}_{d}(t)$ given by (54). By (44), the optimal sustainable rent over $[0, T]$ is also a slowly changing function $\widetilde{R}_{d}(t)=\left(\alpha \tilde{m}_{d}(t)-\beta \tilde{m}_{d}^{2}(t) / 2\right) \hat{s}-c \tilde{m}_{d}(t)-u_{0}$, such that $\tilde{R}_{A}<\widetilde{R}_{d}(t)<R_{\infty}$, where $R_{\infty}$ is given by (45). Correspondingly, we obtain by (56) that

$$
\tilde{R}_{A} / r-V(T)<\tilde{I}(T)<R_{\infty} / r-V(T)
$$

Theorem 6. If (28) holds, $r T \ll<1, \alpha \mu \hat{m} T<<1$, and $f^{\prime} \ll<$, then the reduction of the landlord discounted profit (49) during one contract (compared to the case with no delay) is

$$
J_{d}-J \approx-\frac{\alpha^{3}}{\beta^{2}}\left(1-\frac{c \mu}{g_{0}}\right)^{2} \frac{g_{0} e^{-r T} \Delta^{2}}{6}
$$

and the reduction in the total discounted profit $(1)$ over $[0, \infty)$ is

$$
\tilde{I}_{d}-\tilde{I} \approx-\frac{\alpha^{3}}{\beta^{2}}\left(1-\frac{c \mu}{g_{0}}\right)^{2} \frac{g_{0} \Delta^{2}}{6\left(e^{r T}-1\right)} .
$$

The optimal contract duration is approximately $T^{*}=\operatorname{argmax} V(T)$.

The obtained approximate formulas (54)-(58) for the optimal trajectories in the case with delay demonstrate that the qualitative dynamics of the landlord-tenant problem remain unchanged and the 
sustainable soil quality is approximately the same as in the case with no-delay, however, the landlord experiences a profit reduction determined by (58).

6. Concluding remarks. In order to explore agricultural, economic, and social processes that determine the landlord-tenant relationship, we construct and investigate a hierarchical economicenvironmental model and provide its qualitative analysis. First, the steady-state analysis of the model shows the existence of a sustainable solution for the infinite-horizon landlord problem in specific ranges of model parameters. This solution delivers the best possible environmental quality. Next we provide a dynamic qualitative analysis of the constructed optimization model. Because of nonlinear nature of the problem, we have to use approximate solution techniques to complete this analysis. We show that there exists an initial condition for the soil quality, which is the same for all periods and delivers a periodic solution to the tenant's problem. We also prove that this periodic solution asymptotically attracts other solutions for different initial conditions.

Our analytic findings lead to some interesting economic outcomes and policy recommendations. First of all, the qualitative behavior of the optimal trajectories is affected to a greater extent by environmental and production parameters and initial conditions rather than by the end-of-horizon and delay effects. We identify the range of model parameters when the optimal solution coincides with the first-best (environmentally and socially efficient) solution. It happens when the profitability of the tenant's investment is small and the discount rate is essential. In particular, it means that no holdup problem emerges within this parameter range. Therefore, the holdup problem is possible but avoidable in our model settings. A similar conclusion was obtained by Che and Sakovics (2004) for a discrete-time dynamic problem. However, our impact of the discount rate on the severity of a holdup problem differs from Che and Sakovics (2004) because of the different definition of the first-best socially efficient solution: this solution is not subjected to the discount rate as in Che and Sakovics (2004).

Qualitative properties of our landlord-tenant model, including the holdup problem, can be quite different in cases when the investment profitability parameter is not small. However, then the developed approximation technique fails and nonlinearity of the model does not allow for even simplified approximate analysis of this problem.

With respect to the design of a contract, the results of our study advise the landlord to treat tenants who can buy mineral fertilizer differently from tenants who cannot. If the tenants do not have access to markets, the holdup problem is less likely as shown in this work. However, if tenants have the option to buy mineral fertilizers, they can improve agricultural productivity without improving the soil fertility. 
Then, the emergence of the holdup problem is more likely, so a landlord may be advised to specify in the lease contract that the tenant uses the organic fertilizer.

Acknowledgements. The authors express their sincere gratitude to the Editor Catherine Roberts and two anonymous reviewers for careful reading of the paper and valuable comments. This paper was partially written when Hritonenko and Yatsenko were visiting Goetz and Xabadia in Girona in 2012 and 2013, and the authors are thankful to the University of Girona (Spain) for the financial support of these visits. The work was supported by the NSF DMS grant 1009197 and the Ministerio de Ciencia e Innovación grant Econ2010-17020.

\section{REFERENCES}

A. Abdulai, V. Owusu, and R. Goetz [2011], Land tenure differences and investment in land improvement measures: Theoretical and empirical analyses, Journal of Development Economics 96, 66-78.

A. Akhter, A. Abdulai, and R. Goetz [2012], Impacts of Tenancy Arrangements on Investment and Efficiency: Evidence From Pakistan, Agricultural Economics 43, 1-13.

T. Besley [1995]. Property Rights and Investment Incentives: Theory and Evidence from Ghana, Journal of Political Economy 103, 903-937.

T. Bréchet, N. Hritonenko, and Y. Yatsenko [2013]. Adaptation and mitigation in long-term climate policy, Environmental and Resource Economics 55, 217-243.

M.R. Caputo and D. Lueck [2003], Natural Resource Exploitation under Common Property Rights, Natural Resource Modeling 16, 39-67.

Y.-K. Che and J. Sakovics [2004]. A Dynamic Theory of Holdup, Econometrica 72, 1063-1103.

C. Corduneanu [1991]. Integral Equations and Applications. Cambridge University Press, Cambridge, USA.

P. Dubois [2008]. Sharecropping, in (S. Durlauf and L. Blume, eds.), The New Palgrave Dictionary of Economics. Second Edition. Palgrave, Macmillan. http://www.dictionaryofeconomics.com/article?id= pde2008_S000111 (accessed June 1, 2014) 
F. Gul [2001]. Unobservable Investment and the Hold-up Problem, Econometrica 69, 343-376.

S. Guriev and D. Kvasov [2005]. Contracting on Time, American Economic Review 95, 1369-85.

R. Fraser [2004]. On the Use of Targeting to Reduce Moral Hazard in Agri-environmental Schemes, Journal of Agricultural Economics 55, 525-540.

O. Hart and J. Moore [2007]. Incomplete Contracts and Ownership: Some New Thoughts, American Economic Review 97, 182-186.

N. Hritonenko and Y. Yatsenko [2005]. Turnpike Properties of Optimal Delay in Integral Dynamic Models, Journal of Optimization Theory and Applications 127, 109-127.

N. Hritonenko and Y. Yatsenko [2006]. Optimization of Harvesting Return from Age-structured Population, Journal of Bioeconomics 8, 167-179.

N. Hritonenko and Y. Yatsenko [2012]. Energy Substitutability and Modernization of Energy-consuming Technologies, Energy Economics 34, 1548-1556.

N. Hritonenko and Y. Yatsenko [2013]. Mathematical Modeling in Economics, Ecology and the Environment, 2nd Edition. Springer, New York/Berlin.

N. Hritonenko, Y. Yatsenko, R. Goetz, and A. Xabadia [2012] Optimal Harvesting in Forestry: Steadystate Analysis and Climate Change Impact, Journal of Biological Dynamics 7, 41-58.

H.G. Jacoby and G. Mansury [2008]. Land Tenancy and Non-contractible Investment in Rural Pakistan, Review of Economic Studies 75, 763-788.

B. Jovanovic and Y. Yatsenko [2012]. Investment in Vintage Capital, Journal of Economic Theory 147, 551-569.

K.L.Judd and S.-M. Guu [1997]. Asymptotic Methods for Aggregate Growth Models, Journal of Economic Dynamics and Control 21, 1025-1042.

M. Khan and S Rashid [1982]. Approximate Equilibria in Markets with Invisible Commodities, Journal of Economic Theory 28, 82-101.

W. Kirk and M. Khamsi [2001]. An Introduction to Metric Spaces and Fixed Point Theory. John Wiley, New York. 
A. Kolmogorov and S. Fomin [1975]. Introductory Real Analysis. Dover Publications, New York.

E. Lichtenberg [2007]. Tenants, Landlords, and Soil Conservation, American Journal of Agricultural Economics 89, 294-307.

P. Rey and B Salanie [1990]. Long-term, Short-term and Renegotiation: On the Value of Commitment in Contracting, Econometrica 58, 597-619.

M.S. Santos [1994]. Smooth Dynamics and Computation in Models of Economic Growth, Journal of Economic Dynamics and Control 18, 879-895.

S. Sethi and G. Thompson [2000]. Optimal Control Theory - Applications to Management Science and Economics. Springer, New York/Berlin.

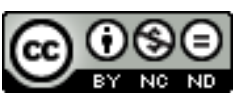

Modeling of a landlord-tenant agricultural system in the environmental context is licensed under a Creative Commons AttributionNonCommercial-NoDerivatives 4.0 International License. Based on a work at https://doi.org/10.1111/nrm.12051. 